\title{
COMPARAÇÃO ENTRE OS MODELOS DE COMBUSTÃO WIEBE E FRACTAL PARA SIMULAÇÃO COMPUTACIONAL DE UM MOTOR FLEX OPERANDO COM DIFERENTES MISTURAS DE GASOLINA- ETANOL
}

\author{
Tadeu Cavalcante Cordeiro de Melo ${ }^{1}$, Guilherme Bastos Machado ${ }^{1}$ e Filipe Augusto Serrão \\ Matias $^{2}$ \\ ${ }^{1}$ CENPES - PETROBRAS \\ ${ }^{2}$ Universidade Federal do Rio de Janeiro (UFRJ)
}

E-mails: tcm@petrobras.com.br, machadogb@petrobras.com.br, fasmatias@gmail.com

\section{RESUMO}

A modelagem da combustão em motores é considerada uma questão complexa na área automotiva. Ainda são necessários desenvolvimentos adicionais para melhorar a simulação do desempenho e das emissões do motor com o uso de diferentes combustíveis. Para estudar a influência de diferentes misturas de gasolina-etanol em um motor do tipo FLEX, foram feitas simulações computacionais utilizando os modelos de combustão Wiebe e Fractal, com o de transferência de calor de Woschini. Após ajustes de parâmetros dos modelos, foi possível validar as simulações com o uso de dados experimentais de dinamômetro de motor. Pressão do cilindro, IMEP e dados de emissões foram calculados para diferentes misturas de gasolina e etanol em diferentes condições de operação do motor. Os resultados das simulações com o modelo Fractal foram comparados aos obtidos com o uso do modelo de combustão de Wiebe, mostrando vantagens e limitações de cada um para simular um motor FLEX com diferentes misturas de gasolina e etanol.

\section{INTRODUÇÃO}

Uma gasolina comercial pode conter mais de 400 diferentes tipos de hidrocarbonetos em sua composição, cada um deles pode evaporar em diferentes temperaturas e apresentar diferentes valores de velocidades de frente de chama. Dessa forma a modelagem do processo de combustão de gasolina é considerada nos dias atuais um tema complexo.

A adição de etanol na gasolina vem sendo adotada em alguns países como forma de introduzir o uso de combustíveis renováveis, normalmente com percentual de até $15 \%$ v/v. No Brasil, a adição de etanol anidro à gasolina pode variar de $18 \%$ a $25 \%$ v/v e existem os veículos FlexFuel que rodam com misturas de 0 a $100 \%$ de etanol hidratado na gasolina nacional. Gerando complexidade aos estudos de modelagem de combustão. $[1,2,3]$.

Melo et al.[2] implementaram um modelo computacional para o estudo da combustão de um motor Flex operando com gasolina, etanol hidratado e GNV (gás natural veicular). O trabalho utilizou a modelagem de combustão de uma zona, proposta por Wiebe e foram obtidos resultados satisfatórios de simulação da curva de pressão e IMEP (pressão média efetiva 
indicada) em relação aos resultados experimentais. Como continuação desse trabalho, Melo et al. [3] propuseram o uso de um modelo Wiebe de duas zonas para modelar a combustão de um motor Flex operando com gasolina com 25\% de etanol e diferentes misturas com etanol hidratado. Os resultados de curva de pressão e IMEP foram satisfatórios, porém com relação às emissões houve a necessidade de ajustes individuais para cada tipo de poluente.

Foi encontrado na literatura apenas um pequeno número de trabalhos de modelagem de motores Flex, sendo assim esse trabalho propõe o uso do modelo de combustão Fractal para simulação do mesmo motor Flex e dos mesmos combustíveis usados no trabalho de Melo et al.[3]. Serão mostradoas as vantagens do modelo Fractal e suas limitações em relação ao modelo do tipo Wiebe adotado nos trabalhos anteriores.

\section{TEORIA}

Nos itens seguintes será detalhada a teoria aplicada na construção do simulador do motor FLEX, tanto para o modelo Fractal quanto para o modelo Wiebe de duas zonas.

\subsection{Coeficiente de transferência de calor}

Para calcular a perda de calor pela parede, foi utilizado a lei de resfriamento de Newton para ambos os modelos (Wiebe e Fractal), dada pela equação 1 [2, 3, 4].

$$
Q_{w}=A_{i} \cdot h_{\text {woschni }}\left(T_{c}-T_{w}\right)
$$

Onde: $\mathrm{Q}_{\mathrm{w}}$ é o calor transferido pela parede; $\mathrm{A}_{\mathrm{i}}$ é a área da câmara de combustão em contato com o gás; $\mathrm{T}_{\mathrm{c}}$ é a temperatura média no interior do cilindro; $\mathrm{T}_{\mathrm{w}}$ é a temperatura média da parede do cilindro.

Melo et al [3] calcularam o coeficiente de transferência de calor $\left(h_{\text {Woschni }}\right)$ como recomendado por Woschni [5].

$$
h_{\text {Woschni }}=0,013 D^{0,2} P_{c}^{0,8} T_{c}^{0,53} v_{g}^{0,8}
$$

Onde: $P_{c}$ e $T_{c}$ são a pressão e a temperatura média no interior do cilindro; $\mathrm{D}$ é o diâmetro do cilindro; e $v_{g}$ é a velocidade do gás na válvula de admissão.

Com o objetivo de melhorar a precisão do cálculo do coeficiente de transferência de calor, a empresa AVL criou a equação 3, que foi baseada no trabalho de Wimmer et al [6] e Schubert et al [7] e foi usada na modelagem do presente artigo.

$$
\mathrm{h}_{\mathrm{AVL}}=0.013 \mathrm{D}^{0.2} \mathrm{P}_{\mathrm{c}}{ }^{0.8} \mathrm{~T}_{\mathrm{c}}{ }^{0.53}\left(\mathrm{C}_{4}\left(\mathrm{~d}_{\mathrm{in}} / \mathrm{D}\right)^{2}\left|\mathrm{v}_{\mathrm{g}}\right|\right)^{0.8}
$$

Onde: $\mathrm{C}_{4}$ é uma constante igual a 14,$0 ; D$ é o diâmetro do cilindro; $d_{i n}$ é o diâmetro interno do tubo conectado à válvula de admissão.

Para usar a equação 1 é necessário a informar a temperatura média da parede. Essa temperatura não é simples de se medir experimentalmente, havendo a necessidade de instalação de um termopar especial no interior do cilindro. Neste trabalho foi utilizado como temperatura média da parede do cilindro o valor de $120^{\circ} \mathrm{C}$. Este valor foi baseado em experimentos realizados anteriormente por Melo et al. [2], que estimaram essa temperatura a partir da temperatura de arrefecimento do motor e com base na experiência da AVL em desenvolvimento e simulação de motores. 
1.2. Modelos de combustão

\subsubsection{Modelo de Wiebe}

A equação 4 do modelo de Wiebe de uma zona calcula a fração de massa de combustível queimado em função do ângulo do eixo do virabrequim, $\operatorname{MFB}(\theta)$ $[2,3,4]$ e está representada graficamente na figura 1.

$$
\operatorname{MFB}(\theta)=1-\exp \left(-a\left(\frac{\theta-\theta_{i}}{\Delta \theta}\right)^{m+1}\right)
$$

Os parâmetros $a$ e $m$ devem ser ajustados para adequar a curva às condições de geometria do motor e do combustível utilizado.

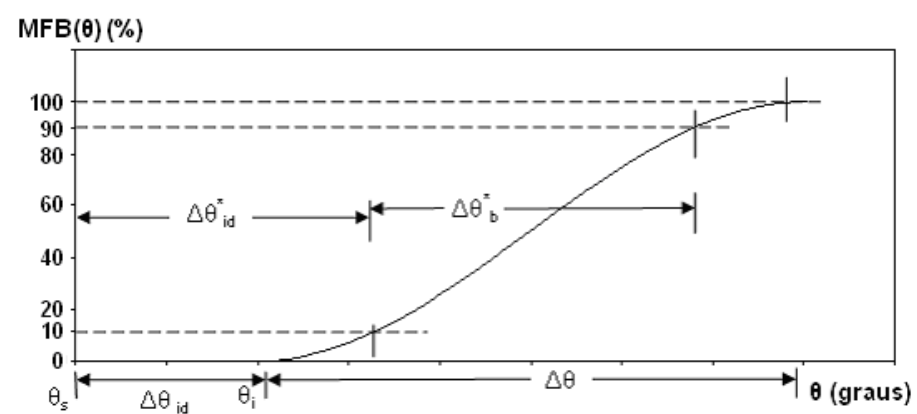

Figura 1: Fração de massa de combustível queimada do modelo wiebe

Onde: $\theta_{\mathrm{s}}$ é o ângulo da centelha da vela; $\theta_{\mathrm{i}}$ é ângulo de início da liberação de calor; $\Delta \theta_{\text {id }}$ é o atraso referente a liberação de calor; $\Delta \theta^{*}{ }_{\text {id }}$ é o intervalo angular total do atraso de ignição; $\Delta \theta^{*}$ b é a duração da combustão encontrada experimentalmente $(10 \%$ a $90 \%$ do $\operatorname{MFB}(\theta)) ; \Delta \theta$ é a duração total da combustão representada pela curva de Wiebe.

Para ajustar a equação 4 são necessários os dados de entrada: $\theta_{\mathrm{i}}, \Delta \theta$. Experimentalmente a duração total da curva de Wiebe é considerada entre $1 \%$ e $99 \%$ de massa de combustível queimada (MFB).

O modelo de Wiebe duas zonas, usado por Melo et al. [3], calcula a fração de massa de combustível queimada pela equação 4. Apesar da pressão do cilindro ser considerada constante para a região de massa queimada e não queimada, a temperatura é diferente. A conservação de energia pode ser aplicada para as massas queimada (b) e não queimada (unb), resultando nas equações 5 e 6 [8].

$\frac{\mathrm{dm}_{\mathrm{b}} \mathrm{u}_{\mathrm{b}}}{\mathrm{d} \theta}=-\mathrm{Pc} \frac{\mathrm{dV}_{\mathrm{b}}}{\mathrm{d} \theta}+\frac{\mathrm{dQ}_{\text {fuel }}}{\mathrm{d} \theta}-\sum \frac{\mathrm{dQ}_{\mathrm{w}, \mathrm{b}}}{\mathrm{d} \theta}+\mathrm{h}_{\mathrm{b}} \frac{\mathrm{dm}_{\mathrm{b}}}{\mathrm{d} \theta}-\mathrm{h}_{\mathrm{BB}, \mathrm{b}} \frac{\mathrm{dm}_{\mathrm{BB}, \mathrm{b}}}{\mathrm{d} \theta}$

$\frac{\mathrm{dm}_{\text {unb }} \mathrm{u}_{\text {unb }}}{\mathrm{d} \theta}=-\mathrm{Pc} \frac{\mathrm{dV}_{\mathrm{unb}}}{\mathrm{d} \theta}-\sum \frac{\mathrm{dQ}_{\mathrm{w}, \text { unb }}}{\mathrm{d} \theta}-\mathrm{h}_{\mathrm{unb}} \frac{\mathrm{dm}_{\text {unb }}}{\mathrm{d} \theta}-\mathrm{h}_{\mathrm{BB}, \text { unb }} \frac{\mathrm{dm}_{\mathrm{BB}, \text { unb }}}{\mathrm{d} \theta}$

Onde o termo $\frac{\mathrm{h}_{\mathrm{unb}} \cdot \mathrm{dm} \mathrm{m}_{\text {unb }}}{\mathrm{d} \theta}$ considera o fluxo de entalpia da região de gases não queimados para a região de gases queimados devido à conversão de uma carga nova de mistura ar-combustível em produtos da combustão. Vale ressaltar que não foi considerado o fluxo de calor entre as duas zonas. 
O modelo considera que a soma das taxas de variação de volume de cada uma das zonas é igual à variação total de volume no cilindro assim como apresentado pela equação 7 . Outra relação considerada, e explicitada pela equação 8 , é que o volume total instantâneo no interior do cilindro é igual à soma do volume de massa queimada e não queimada [8].

$$
\begin{aligned}
& \frac{\mathrm{dV}_{\mathrm{b}}}{\mathrm{d} \theta}+\frac{\mathrm{dV}_{\text {unb }}}{\mathrm{d} \theta}=\frac{\mathrm{dV}}{\mathrm{d} \theta} \\
& V_{b}+V_{\text {unb }}=V
\end{aligned}
$$

\subsubsection{Modelo Fractal}

O modelo fractal aplicado nesse trabalho, tem como objetivo modelar a taxa de queima da massa no interior do cilindro. Esse cálculo é então utilizado no modelo de queima duas-zonas já mencionado no item 1.2.1 (equações 4 a 8). A principal diferença do modelo Fractal para o modelo Wiebe é que o modelo Fractal busca calcular a taxa de calor liberado a partir da teoria de superfície fractal e escoamento turbulento, não sendo necessários os dados das curvas de pressão experimentais para cada caso que se deseja simular. Por outro lado, no modelo Wiebe, para cada taxa de calor liberado na combustão em cada condição de operação do motor e misturas de combustíveis são necessários ajustes dos parâmetros $a$ e $m$, baseados em dados experimentais.

O modelo fractal leva em conta a influência dos parâmetros: geometria da câmara de combustão, localização da vela, composição do gás no cilindro, e turbulência do fluido. Neste modelo, a frente de chama começa como uma pequena esfera de superfície lisa e esbelta. Durante o progresso da combustão, esta superfície é enrugada devido à turbulência. Esta consideração é uma boa aproximação para o modelo de queima desde que a velocidade de rotação do motor não seja muito elevada, neste caso a frente de chama passa a se unir e a geometria de uma esfera enrugada deixa de ser uma boa aproximação.

Damköhler [9] propôs o cálculo da taxa de queima da massa pela equação 9.

$$
\frac{d m_{b}}{d t}=\rho_{u} A_{T} S_{L}=\bar{\rho}_{u} A_{L} S_{T}
$$

Onde a área $A_{T}$ é a área da frente de chama enrugada pela turbulência; $A_{L}$ é considerado a área de uma frente de chama teórica caso não houvesse turbulência (escoamento laminar); $S_{L}$ é a velocidade laminar da frente de chama; $S_{T}$ é a velocidade turbulenta da frente de chama; $\rho_{u}$ é a massa específica pontual da mistura na câmara de combustão; e $\bar{\rho}_{u}$ é a massa específica média da mistura.

$\mathrm{Na}$ figura 2 é apresentado um esquema da frente de chama turbulenta exemplificando as velocidades turbulentas e laminares, assim como suas respectivas áreas de chama. 
Figura 2: Escoamento turbulento entre duas placas

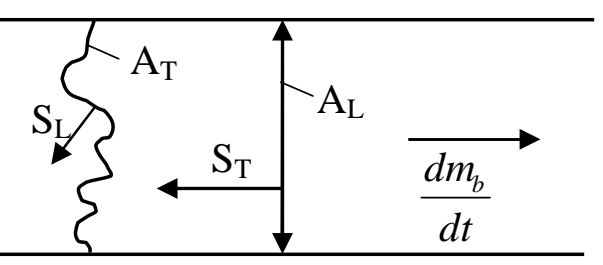

Como a massa específica $\rho_{u}$ é considerada constante, então $\rho_{u}=\bar{\rho}_{u}$, sendo assim a relação pode ser expressa pela equação 10 [9]:

$$
\frac{A_{T}}{A_{L}}=\frac{S_{T}}{S_{L}}
$$

Substituindo a equação 10 na equação 9 obtém-se a seguinte equação:

$$
\frac{d m_{b}}{d t}=\bar{\rho}_{u} A_{L} S_{T}=\rho_{u} \frac{A_{T}}{A_{L}} A_{L} S_{L}
$$

A taxa de queima da massa pode ser expressa como uma função da velocidade laminar de queima que é uma propriedade do combustível (equação 11). Para isso deve-se saber a razão entre as áreas laminar e turbulenta.

Os mecanismos responsáveis pelo enrugamento da superfície da frente de chama ainda não são completamente conhecidos, porém, para esse trabalho, a frente de chama será simplificada por uma superfície esférica única apenas enrugada devido ao fenômeno da turbulência. Com essa consideração pode-se afirmar que a turbulência não é intensa o suficiente para que diferentes pontos da frente de chama se unam, tornando o modelo de combustão fractal válido.

Damköhler [9] propôs a equação 12 para cálculo da área da superfície fractal, quando as escalas da superfície fractal se encontram entre $l_{\min }$ e $l_{\max }$.

$$
A_{T}=A_{L}\left(\frac{l_{\text {max }}}{l_{\text {min }}}\right)^{D_{3}-2}
$$

Onde $D_{3}$ é conhecido como dimensão fractal.

Como o enrugamento da frente de chama foi assumido como gerado apenas pela turbulência, então $l_{\min }, l_{\max }$ e $D_{3}$ serão encontrados nas equações de turbulência do escoamento dentro do cilindro.

\section{Turbulência}

Existem algumas teorias na literatura que modelam o escoamento turbulento. Para simular o modelo de combustão fractal, foi usado o modelo de turbulência de duas equações $K-k$, que é representado pelas seguintes equações [10]:

$$
\begin{aligned}
& \frac{d K}{d t}=\frac{1}{2} \dot{m}_{i n} u_{i n}^{2}-P_{T}+K \frac{\dot{m}_{e x}}{m}+K \frac{\dot{\rho}_{u}}{\rho_{u}} \\
& \frac{d k}{d t}=P_{T}-m \varepsilon+k \frac{\dot{m}_{e x}}{m}+k \frac{\dot{\rho}_{u}}{\rho_{u}}
\end{aligned}
$$




$$
\begin{aligned}
& P_{T}=0.3307 c_{t} \frac{K}{L_{I}} \sqrt{\frac{k}{m}} \\
& K=\frac{1}{2} m U_{f}^{2} \\
& k=\frac{3}{2} m u^{\prime 2} \\
& \mathcal{E}=\frac{u^{\prime 3}}{L_{I}}
\end{aligned}
$$

Onde $u^{\prime}$ é a intensidade turbulenta; $K$ é a energia cinética do escoamento médio; $\dot{m}_{e x}$ é a taxa de exaustão do escoamento; $k$ é a energia cinética do escoamento turbulento e $\varepsilon$ é a sua taxa de dissipação; $P_{T}$ é a produção de turbulência (representa a energia transferida do escoamento principal para o escomento turbulento); e $c_{t}$ é o parâmetro para ajustar a produção de turbulência (constante de produção de turbulência).

Kolmogorov encontrou que a menor escala do escoamento turbulento é dada pela equação 19.

$$
l_{\text {min }}=\left(\frac{v^{3}}{\xi}\right)^{1 / 4}
$$

Onde: $v$ é a viscosidade cinemática da mistura não queimada; $\xi$ é a taxa de dissipação viscosa, definida pela equação 20.

$$
\xi=\left(\frac{\left(u^{\prime}\right)^{3}}{l_{\max }}\right)
$$

Sendo que:

$$
l_{\max }=c_{l} H
$$

Onde $H$ é a distância entre o cabeçote e o pistão em cada dado instante; e $c_{l} \mathrm{o}$ Parâmetro Turbulento de Escala de Comprimento, ajustado posteriormente.

A dimensão fractal $\left(D_{3}\right)$ depende basicamente da velocidade laminar $\left(S_{L}\right)$ e da intensidade turbulenta $\left(u^{\prime}\right)$ [11], sendo dado pela equação 22.

$D_{3}=\frac{2.35 u^{\prime}+2.05 S_{L}}{u^{\prime}+S_{L}}$

O modelo fractal descrito até o momento é uma boa aproximação para equacionar a taxa de queima de uma chama bem desenvolvida. Porém, não se pode usar essa teoria sem que sejam feitos ajustes para os estágios iniciais de desenvolvimento da chama e para a queima quando a frente de chama começa a entrar em contato com a parede do cilindro.

\section{Fase inicial e média da combustão}

$\mathrm{O}$ inicio da combustão é um fenômeno bastante complexo. De acordo com North et al. [11] esse estágio começa pela formação de plasma seguida pela evolução da chama de kernel. O software BOOST inclui um parâmetro $\left(\mathrm{c}_{\mathrm{ign}}\right)$ que é responsável por ajustar a evolução da chama de kernel, que em geral é 
em torno de 200ms. Durante essas fases a velocidade de propagação da chama é muito elevada, até atingir aproximadamente $2 \mathrm{~mm}$ de diâmetro (fim da evolução da chama de kernel). Os estágios da chama até o fim da evolução da chama de kernel não são incluídos no calculo computacional. Este cálculo começa apenas após essa etapa, e como condição inicial da chama, é considerado uma superfície esférica esbelta e lisa de $2 \mathrm{~mm}$ de diâmetro. $\mathrm{O}$ processo de enrugamento da frente de chama é considerado proporcional ao raio da frente de chama (em relação ao seu ponto de origem - vela) e à turbulência do escoamento. A equação 23 foi proposta para o calculo adimensional da taxa de enrugamento da superfície [12].

$$
\omega_{w r}=\frac{r_{f}}{r_{f, r e f}} \frac{n}{n_{r e f}}
$$

Onde: $\mathrm{n}_{\text {ref }}$ é uma velocidade de referência do motor no valor de 1000rpm; $\mathrm{n}$ é a velocidade em rpm do motor; $r_{f}$ é o raio instantâneo do frente de chama; $r_{f, r e f}$ (relação do raio de ignição) é um parâmetro referente a um raio de $1 \mathrm{~cm}$ que deve ser ajustado no software pelo usuário. De acordo com a AVL, em sua experiência em modelagem computacional de motores, o ajuste desse parâmetro para $1 \mathrm{~cm}$ é um bom valor para a maioria dos motores.

A equação 22 não é uma boa aproximação para a modelagem da combustão fractal, principalmente devido à particularidade do início da combustão. Por esse motivo ela foi modificada para levar em conta esse estágio (equação 25)

$$
D_{3}=\frac{D_{3, \max } u^{\prime}+D_{3, \min } S_{L}}{u^{\prime}+S_{L}}
$$

Onde: $D_{3, \min }=2.05 ; D_{3, \max }=D_{3, \text { min }}\left(1-w_{1}\right)+2.35 w_{1} ; w_{1}=1-\exp \left(-\omega_{w r}\right)$

No início do cálculo computacional da combustão, $\mathrm{D}_{3}$ esta muito próximo de seu valor mínimo $\mathrm{D}_{(3, \mathrm{~min})}$, que será sempre maior que 2 . Este fato deve ser suficiente para compensar a fase inicial da combustão que não está inclusa no calculo computacional.

\section{Combustão próxima à parede}

O modelo fractal descrito até então não é apropriado para descrever a combustão próxima da parede [13]. A equação 25 foi proposta para modelar a taxa de queima próximo da parede [12].

$$
\left(\frac{d m_{b}}{d t}\right)_{\text {wall-combustion }}=\frac{m-m_{b}}{\tau}
$$

Onde $m-m_{b}$ é a massa de gás não queimado e $\tau$ é a escala de tempo característico.

A taxa total de queima próxima da parede pode ser descrita pela equação 26 .

$$
\left(\frac{d m_{b}}{d t}\right)_{\text {overall }}=\left(1-w_{2}\right)\left(\frac{d m_{b}}{d t}\right)_{\text {fractal }}+w_{2}\left(\frac{d m_{b}}{d t}\right)_{\text {wall-combustion }}
$$


A equação acima mostra que a transição da combustão do modelo Fractal para o modelo de queima próxima à parede é feita gradualmente, com o uso do fator $w_{2}$. A transição começa a ocorrer quando o tempo de transição é atingido, ou seja, quando o primeiro ponto da frente de chama atinge a parede do pistão.

A escala de tempo característico, equação 27, é calculada assumindo que a taxa de queima da combustão próxima à parede é a mesma que a do modelo fractal.

$$
\tau=\frac{\left(m-m_{b}\right)_{t r}}{\left(\rho_{u} A_{T} S_{L}\right)_{t r}}
$$

Enquanto $\tau$ é mantido constante durante a combustão próxima à parede, o fator de peso $w_{2}$ aumenta linearmente com o tempo como função da massa não queimada.

$$
w_{2}=1-\frac{\left(m-m_{b}\right)}{\left(m-m_{b}\right)_{t r}}
$$

\subsection{Emissões}

Ambos os modelos (Wiebe e Fractal) utilizaram os mesmos cálculos de emissões do programa BOOST descritos a seguir.

\subsubsection{Formação de NOx}

A teoria utilizada pelo software BOOST para calcular as emissões de NOx é baseada no modelo de formação de NO de Pattas et al. [14]. Este modelo inclui três equações do mecanismo de Zeldovich [4], além de três outras equações para calcular a formação de $\mathrm{N}_{2} \mathrm{O}$. As taxas de reações ( $\mathrm{R} 1$ a R6) estão descritas detalhadamente no trabalho de Melo et al. [3]

A taxa final de produção/destrução de NO pode ser calculada pela equação 30 .

$$
\frac{\mathrm{d}[\mathrm{NO}]}{\mathrm{dt}}=\mathrm{NO} \_\mathrm{POST} \times \mathrm{NO} \_\mathrm{MULT} \times 2,0 \times\left(1-\beta^{2}\right) \frac{\mathrm{R}_{1}}{1+\varepsilon \times \mathrm{AK}_{1}}
$$

Onde $\varepsilon$, AK1 e AK2 são calculados pelas equações 31, 32 e 33.

$$
\begin{aligned}
& \varepsilon=\frac{[\mathrm{NO}]}{[\mathrm{NO}]_{\mathrm{eq}}} \times \frac{1}{\mathrm{NO} \_\mathrm{POST}} \\
& \mathrm{AK}_{1}=\frac{\mathrm{R}_{1}}{\mathrm{R}_{2}+\mathrm{R}_{3}} \\
& \mathrm{AK}_{2}=\frac{\mathrm{R}_{4}}{\mathrm{R}_{5}+\mathrm{R}_{6}}
\end{aligned}
$$

O software da AVL utiliza dois parâmetros que devem ser ajustados pelo usuário para acertar as emissões de NOx. Estes parâmetros são o NO_POST e o NO_MULT. O primeiro parâmetro afeta tanto a formação quanto a oxidação após o período de combustão (expansão e exaustão), e o segundo ajusta a formação de NO no período de combustão. 


\subsubsection{Formação de $\mathrm{CO}$}

Para estimar a formação de monóxido de carbono $(\mathrm{CO})$, foram utilizados mecanismos baseados em Onorati et al [15] e em Raggi et al. [16].

A formação/destruição de CO pode ser calculada pela equação 34 [15, 16].

$$
\frac{\mathrm{d}[\mathrm{CO}]}{\mathrm{dt}}=\text { CO_MULT }\left(\mathrm{R}_{7}+\mathrm{R}_{8}\right)\left(1-\frac{[\mathrm{CO}]}{[\mathrm{CO}]_{\mathrm{e}_{\mathrm{q}}}}\right)
$$

Onde: "eq" = equilíbrio químico; CO_MULT = parâmetro a ser ajustado.

\subsubsection{Emissões de $H C$}

Em um motor a combustão interna, há diferentes fontes dos hidrocarbonetos responsáveis por compor as emissões de HC. As duas fontes consideradas mais importantes são: - Parte do combustível na câmara de combustão se aloca no volume entre o pistão e a câmara de combustão, onde a chama não se propaga; - Parte do combustível vaporizado se difunde para o óleo lubrificante que se encontra na parede do pistão, durante a admissão e compressão, e não é queimado, sendo expelido na fase de exaustão. Para a simulação das emissões de HC foi utilizada uma correlação semi-empírica baseada no trabalho de Lavoie et al. [17] e que pode ser encontrado no trabalho de Melo et al. [3].

\section{DADOS EXPERIMENTAIS}

Para permitir a adequada calibração do modelo computacional desenvolvido nesse trabalho, foram necessários vários dados experimentais. O trabalho de Melo et al. [3] contém os dados experimentais necessários para a calibração e validação dos modelos computacionais propostos para esse trabalho e serão re-apresentados a seguir [3].

\subsection{Combustíveis}

Os combustíveis usados para a realização dos experimentos e das simulações computacionais foram: - gasolina comercial com 25\% de etanol anidro (H0); - 100\% de etanol hidratado (H100); - misturas de $30 \%$ v/v (H30), $50 \%$ v/v (H50) e $80 \%$ v/v (H80) de etanol hidratado (H100) e gasolina E25 (H0). As principais propriedades dos combustíveis necessárias para uso nas simulações estão listadas na tabela 1.

Tabela 1 - Principais propriedades dos combustíveis necessárias para ajustar o software de simulação

\begin{tabular}{|c|c|c|c|c|c|}
\hline $\begin{array}{c}\text { Propriedades dos combustíveis/ } \\
\text { Método ASTM }\end{array}$ & \multicolumn{5}{|c|}{ Etanol hidratado } \\
\cline { 2 - 6 } & $\mathrm{H} 0(\mathrm{E} 25)$ & $\mathrm{H} 30$ & $\mathrm{H} 50$ & $\mathrm{H} 80$ & $\mathrm{H} 100$ \\
\hline Densidade (kg/m $\left.)^{3}\right) / 4052$ & 748,2 & 764,9 & 779,2 & 797,7 & 808,7 \\
\hline MON/ D2700 & 85,1 & 88 & 89,7 & 91,6 & 91,8 \\
\hline Etanol (\% v/v) Calculado & 25,0 & 46,3 & 60,3 & 81,6 & 95,7 \\
\hline Gasolina (\% v/v) Calculado & 75,0 & 52,5 & 37,5 & 15,0 & 0,0 \\
\hline $\mathrm{H}_{2} \mathrm{O}(\%$ v/v) Calculado & 0,0 & 1,2 & 2,2 & 3,4 & 4,3 \\
\hline $\begin{array}{c}\text { Razão ar combustível estequiométrica } \\
\text { Calculado }\end{array}$ & 12,7 & 11,1 & 10,3 & 9,3 & 8,8 \\
\hline PCI (MJ/kg) D5865 & 38,92 & 34,68 & 31,84 & 27,59 & 24,76 \\
\hline RVP (kPa) D5191 & 55,9 & 52,5 & 47,2 & 33,0 & 15,4 \\
\hline
\end{tabular}


Nesse trabalho foi usado o motor modelo Flex 1,4 L cujas principais propriedades geométricas necessárias para uma modelagem do tipo Wiebe estão listadas na tabela 2.

Tabela 2 - Principais características do motor Flex usado

\begin{tabular}{|l|l|}
\hline $\mathrm{N}^{\circ}$ de cilindros & Quatro em série \\
\hline $\mathrm{N}^{\circ}$ de válvulas por cilindro & duas \\
\hline Diâmetro do cilindro & $72.0 \mathrm{~mm}$ \\
\hline Curso do pistão & $84 \mathrm{~mm}$ \\
\hline Diâmetro do pistão & $71.9 \mathrm{~mm}$ \\
\hline Tipo de injeção & PFI \\
\hline Distância entre centros - biela & $128.95 \mathrm{~mm}$ \\
\hline Cilindrada total & $1368 \mathrm{~cm} 3$ \\
\hline Razão de compressão & $10.35: 1$ \\
\hline Torque máximo & $120 \mathrm{Nm}$ a $2250 \mathrm{rpm}$ \\
\hline Potência máxima & $56.8 \mathrm{~kW}$ a $5500 \mathrm{rpm}$ \\
\hline Área de superfície do pistão & $4245 \mathrm{~mm}^{2}$ \\
\hline Área de superfície do cabeçote & $5066 \mathrm{~mm}{ }^{2}$ \\
\hline $\begin{array}{l}\text { Área do cilindro com o pistão no } \\
\text { PMS (Ponto Morto Superior) }\end{array}$ & $68 \mathrm{~mm}{ }^{2}$ \\
\hline $\begin{array}{l}\text { Diâmetro interno da sede da } \\
\text { válvula de admissão }\end{array}$ & $31.3 \mathrm{~mm}$ \\
\hline $\begin{array}{l}\text { Diâmetro interno da sede da } \\
\text { válvula de exaustão }\end{array}$ & $24.2 \mathrm{~mm}$ \\
\hline Folga das válvulas & $0.5 \mathrm{~mm}$ \\
\hline
\end{tabular}

Além dos dados da tabela 2 também foram levantados outros dados do motor para uso na simulação Fractal. Os ângulos de abertura e de fechamento das válvulas de admissão e de escapamento do motor foram medidos pelo CENPES usando equipamentos laser de elevada precisão. As curvas de coeficiente de descarga das válvulas foram levantadas pela empresa AVL em uma bancada de fluxo específica.

A modelagem fractal demandou dados adicionais de geometria do motor, além dos listados na tabela 2, que foram medidos experimentalmente (tabela 3 ).

Tabela 3 - Dados adicionais da geometria do motor para a modelagem fractal

\begin{tabular}{|l|l|}
\hline Tipo da geometria do cabeçote & Esférica \\
\hline Diâmetro da geometria do cabeçote & $58.6 \mathrm{~mm}$ \\
\hline Altura da geometria do cabeçote & $8.7 \mathrm{~mm}$ \\
\hline Tipo de geometria do pistão & Esférico côncavo \\
\hline Profundidade da geometria do pistão & $5.5 \mathrm{~mm}$ \\
\hline Diâmetro da geometria do pistão & $55 \mathrm{~mm}$ \\
\hline $\begin{array}{l}\text { Distância do centro da geometria do pistão em } \\
\text { relação ao centro do pistão }\end{array}$ & $4 \mathrm{~mm}$ \\
\hline $\begin{array}{l}\text { Ângulo entre o centro da geometria do cabeçote } \\
\text { e a vela em relação ao centro do pistão }\end{array}$ & 45 graus \\
\hline Distância da vela ao centro do pistão & $15 \mathrm{~mm}$ \\
\hline
\end{tabular}

\subsection{Procedimentos Experimentais}

Conforme mencionado anteriormente serão listados a seguir um resumo dos principais detalhes do experimento realizado por Melo et al. [3], cujos dados foram usados para calibrar e validar o modelo proposto nesse presente artigo. 
Foi usada uma central eletrônica programável (ECU) fornecida pela empresa MoTec, sendo o avanço otimizado para a condição de máximo torque sem ocorrência de detonação (MBT) para cada condição de operação. O motor foi testado em duas condições de torque (60 Nm e $105 \mathrm{Nm}$ ) e três rotações (1500, 2500 e $3875 \mathrm{rpm})$. As condições de torque $60 \mathrm{Nm}$ foram estequiométricas, ou seja, lambda igual a 1 e as condições de torque de $105 \mathrm{Nm}$ foram com lambda de 0,9. As medições de pressão do cilindro e o cálculo do IMEP (pressão média efetiva indicada) foram feitas com o uso do sistema AVL indimodul, enquanto que as emissões de gases e de etanol não queimado foram medidas com o equipamento AVL SESAM FTIR (Fourier Transform Infrared Analyzer).

O procedimento que foi usado para a realização dos ensaios em motor pode ser descrito de forma resumida como: - Limpar linhas de combustível do banco de provas de motor; - Abastecer combustível de teste e aquecer o motor; - Ajustar ângulo de avanço para o ponto de MBT para cada condição operacional; - Realizar os três ensaios para cada combustível e condição operacional medindo: - Dados do dinamômetro (vazão de ar, consumo de combustível, potência, torque, etc.); - adquirir 300 curvas de pressão do cilindro do motor; - medir as emissões de gases; - Realizar o cálculo das médias de cada variável medida; - Selecionar a curva de pressão do cilindro representativa da condição operacional [3].

\section{SIMULAÇÃ̃O}

O primeiro passo para a criação do modelo de simulação do motor, seja para uso com o modelo do tipo Wiebe usado por Melo et al. [2] seja para o modelo fractal proposto nesse trabalho, é a construção de um modelo geométrico do motor no software de simulação. Para esse trabalho foi usado o software AVL BOOST.

O modelo usa oito parâmetros que são ajustados manualmente no software de simulação, sendo que são recomendados, pelo fabricante, valores iniciais de ajuste para esses parâmetros conforme lista da tabela 6. Devido ao grande volume de dados durante a simulação, para esse trabalho serão apresentados os dados de simulação para a rotação de $3875 \mathrm{rpm}$ para as duas condições de torque testadas (60 Nm e $105 \mathrm{Nm}$ ).

\subsection{Modelo de Combustão de Wiebe}

Para ajustar o modelo de combustão de Wiebe foi necessário ajustar os parâmetros $a$ e m. O ajuste foi feito para cada mistura de combustível e cada condição do motor, buscando equiparar a curva de fração de massa de combustível queimado (MFB) experimental com a simulada pela equação de Wiebe [3].

O ajuste dos parâmetros foi feito mantendo o parâmetro $m=1$ constante para todos os casos enquanto o parâmetro $a$ foi ajustado. Nas tabelas 4 e 5 encontram-se os valores dos parâmetros ajustados por Melo et al [3].

Tabela 4: Parâmetros da Wiebe para 3875rpm à 60Nm

\begin{tabular}{|l|c|c|c|c|c|}
\hline Parameter Fuel & H0 & H30 & H50 & H80 & H100 \\
\hline $\mathrm{a}$ & 3,8 & 4,8 & 5,1 & 5,5 & 3,8 \\
\hline $\mathrm{m}$ & 1 & 1 & 1 & 1 & 1 \\
\hline
\end{tabular}


Tabela 5: Parâmetros da Wiebe para 3875rpm à 105Nm

\begin{tabular}{|l|c|c|c|c|c|}
\hline Parameter Fuel & H0 & H30 & H50 & H80 & H100 \\
\hline $\mathrm{a}$ & 2,9 & 7,3 & 7,3 & 6,5 & 6,4 \\
\hline $\mathrm{m}$ & 1 & 1 & 1 & 1 & 1 \\
\hline
\end{tabular}

\subsection{Modelo de Combustão Fractal}

Os parâmetros para ajustar o modelo de combustão Fractal estão na tabela 7.

Tabela 7 - Parâmetros usados para ajustar o modelo fractal no software de simulação.

\begin{tabular}{|c|c|c|}
\hline PARÂMETRO & $\begin{array}{c}\text { VALORES } \\
\text { INICIAIS } \\
\text { RECOMENDADO } \\
\mathrm{S}\end{array}$ & FUNÇÃO \\
\hline Atraso de Ignição & - & $\begin{array}{l}\text { Especifica o início da } \\
\text { combustão }\end{array}$ \\
\hline Multiplicador da Formação de Ignição $\left(\mathrm{C}_{\text {ign }}\right)$ & 1,0 & \multirow{2}{*}{$\begin{array}{l}\text { Parâmetros para ajustar o } \\
\text { atraso de ignição }\end{array}$} \\
\hline Relação do Raio de Ignição $\left(r_{f, r e f}\right)$ & 0,01 & \\
\hline Constante de Produção de Turbulência $\left(c_{t}\right)$ & 0,2 & \multirow{3}{*}{$\begin{array}{l}\text { Parâmetros para ajustar o } \\
\text { modelo de combustão }\end{array}$} \\
\hline Parâmetro Turbulento de Escala de Comprimento $\left(c_{l}\right)$ & 2 & \\
\hline $\begin{array}{l}\text { Expoente de Densidade Turbulenta de Escala de } \\
\text { Comprimento }\end{array}$ & $-0,33$ & \\
\hline $\begin{array}{l}\text { Fração de Massa para Início da Fase de Combustão } \\
\text { Próxima à Parede }\end{array}$ & 0,5 & \multirow{2}{*}{$\begin{array}{l}\text { Determina o inicio do } \\
\text { modelo de combustão } \\
\text { próximo à parede. }\end{array}$} \\
\hline Expoente LFS & 0,5 & \\
\hline
\end{tabular}

Para facilitar a calibração do modelo fractal, quatro parâmetros tiveram os valores mantidos segundo a recomendação do fabricante do software, um parâmetro foi obtido experimentalmente (avanço de ignição), e três parâmetros foram ajustados.

O modelo Wiebe aplicado no trabalho de Melo et al. [3] precisa de vários parâmetros experimentais do motor incluindo dados de fração de massa de combustível queimado (MFB), que foram calculados a partir da curva de pressão do motor. Dessa forma o modelo fractal apresenta uma importante evolução em relação ao modelo Wiebe, pois não necessita de dados experimentais de combustão.

Os três parâmetros que precisam ser ajustados são: - Multiplicador da Formação de Ignição $\left(c_{i g n}\right)$, Constante de Produção de Turbulência $\left(c_{t}\right)$ e Parâmetro Turbulento de Escala de Comprimento $\left(c_{l}\right)$. Foi desenvolvido então um procedimento para otimizar o ajuste desses parâmetros, sendo o Multiplicador da Formação de Ignição $\left(c_{i g n}\right)$ o primeiro parâmetro a ser ajustado. Ele é responsável pelo ajuste do tempo de atraso entre a centelha e o real início da combustão. Foi necessário o uso da curva experimental de combustão para o etanol hidratado (H100). Enquanto apenas esse parâmetro variava, todos os outros ficaram com os valores iniciais (tabela 7) [8].

O objetivo foi encontrar um simples procedimento para ajustar os parâmetros do modelo fractal de forma a serem aplicados para diferentes misturas de etanol hidratado na gasolina. Alguns valores inicialmente usados para o parâmetro Multiplicador da Formação de Ignição foram: 0,$01 ; 0,5 ; 1 ; 2 ; 4 ; 5 ; 6 ; 7 ; 20$. Para ajustar os ângulos em que ocorre a pressão máxima do cilindro do motor, foram feitas simulações utilizando o modo sem ocorrência de combustão ("motored engine"). 
Comparando os dados da curva de pressão experimental com os dados da curva simulada sem combustão, foi possível determinar o ângulo em que essa curva de pressão começava a se distanciar da curva experimental. O parâmetro Multiplicador da Formação de Ignição foi ajustado para que esse ângulo fosse o mesmo da curva de pressão experimental. A taxa de elevação de pressão nesse momento não precisava ser igual, pois foi ajustada depois pelos parâmetros de turbulência.

Os outros dois parâmetros, Constante de Produção de Turbulência e Parâmetro Turbulento de Escala de Comprimento são usados para ajustar a turbulência da combustão. O parâmetro Constante de Produção de Turbulência é responsável pela taxa de crescimento da intensidade da turbulência $\left(u^{\prime}\right)$ nos estágios iniciais de abertura da válvula de admissão. Por outro lado, o Parâmetro Turbulento de Escala de Comprimento é responsável pela taxa de decaimento da intensidade de turbulência na fase de compressão.

O ajuste desses dois parâmetros foi feito simultaneamente com o uso do combustível H100. Foi criada uma lista com todas as possíveis combinações de cinco diferentes valores variando-os entre 0 e 1 . Depois da conclusão das simulações para todos os casos dessa lista, uma tabela foi criada com os seguintes dados: pressão máxima do cilindro do motor $\left(\mathrm{P}_{\max }\right)$ e ângulo do virabrequim correspondente $\left(\theta_{\mathrm{Pmax}}\right)$. Os dois parâmetros foram ajustados na tentativa de se alcançar simultaneamente os valores experimentais de $P_{\max }$ e $\theta_{P \max }$, sendo o ajuste de $P_{\max }$ priorizado. Em seguida foram usados os dados experimentais do combustível H0 para ajustar o Parâmetro Turbulento de Escala de Comprimento. Os outros parâmetros ficaram constantes, conforme ajustes feitos anteriormente. O principal objetivo do ajuste desse parâmetro foi igualar o valor de $\mathrm{P}_{\max }$ simulado com o experimental.

Para determinar os valores do Parâmetro Turbulento de Escala de Comprimento para outras misturas de etanol, foi feita uma regressão linear usando os valores ajustados desse parâmetro para a gasolina (H0) e para o etanol hidratado (H100), como função do teor de etanol adicionado.

Depois dos ajustes mencionados, que foram realizados para as duas cargas do motor (60 Nm e $105 \mathrm{Nm}$ ), foi possível a criação de duas tabelas com os valores dos parâmetros para o modelo fractal. Os valores para a condição 3875 rpm, 60 Nm e 105 $\mathrm{Nm}$ são apresentados respectivamente nas tabelas 7 e 8 .

Tabela 7 - Valores atribuídos a cada parâmetro do modelo fractal para o caso de $3875 \mathrm{rpm}$ a $60 \mathrm{Nm}$.

\begin{tabular}{|l|c|c|c|c|c|}
\hline \multicolumn{1}{|c|}{ Fuel } & H0 & H30 & H50 & H80 & H100 \\
\hline Parameter & -22 & -28 & -32 & -33 & -34 \\
\hline Atraso de Ignição & 2,7 & 2,7 & 2,7 & 2,7 & 2,7 \\
\hline Multiplicador da Formação de Ignição $\left(C_{\text {ign }}\right)$ & 0,01 & 0,01 & 0,01 & 0,01 & 0,01 \\
\hline Relação do Raio de Ignição $\left(r_{f, r e f}\right)$ & 0,02 & 0,02 & 0,02 & 0,02 & 0,02 \\
\hline Constante de Produção de Turbulência $\left(c_{t}\right)$ & 0,12 & 0,132 & 0,14 & 0,152 & 0,16 \\
\hline Parâmetro Turbulento de Escala de Comprimento $\left(c_{l}\right)$ & $-0,33$ & $-0,33$ & $-0,33$ & $-0,33$ & $-0,33$ \\
\hline $\begin{array}{l}\text { Expoente de Densidade Turbulenta de Escala de } \\
\text { Comprimento }\end{array}$ & 0,2 & 0,2 & 0,2 & 0,2 & 0,2 \\
\hline $\begin{array}{l}\text { Fração de Massa para Início da Fase de Combustão } \\
\text { Próxima à Parede }\end{array}$ & 0,33 & 0,33 & 0,33 & 0,33 & $0 ., 3$ \\
\hline Expoente LFS & & & & \\
\hline
\end{tabular}


Tabela 8 - Valores atribuídos a cada parâmetro do modelo fractal para o caso de $3875 \mathrm{rpm}$ a $105 \mathrm{Nm}$.

\begin{tabular}{|l|c|c|c|c|c|}
\hline \multicolumn{1}{|c|}{ Fuel } & $\mathrm{H} 0$ & $\mathrm{H} 30$ & $\mathrm{H} 50$ & $\mathrm{H} 80$ & $\mathrm{H} 100$ \\
\hline Parameter & -22 & -24 & -28 & -29 & -30 \\
\hline Mutraso de Ignição & 1.3 & 1.3 & 1.3 & 1.3 & 1.3 \\
\hline Relação do Raio de Ignição $\left(r_{f, r e f}\right)$ & 0.01 & 0.01 & 0.01 & 0.01 & 0.01 \\
\hline Constante de Produção de Turbulência $\left(c_{t}\right)$ & 0.01 & 0.01 & 0.01 & 0.01 & 0.01 \\
\hline Parâmetro Turbulento de Escala de Comprimento $\left(c_{l}\right)$ & 0.093 & 0.0912 & 0.09 & 0.0882 & 0.087 \\
\hline $\begin{array}{l}\text { Expoente de Densidade Turbulenta de Escala de } \\
\text { Comprimento }\end{array}$ & -0.33 & -0.33 & -0.33 & -0.33 & -0.33 \\
\hline $\begin{array}{l}\text { Fração de Massa para Início da Fase de Combustão } \\
\text { Próxima à Parede }\end{array}$ & 0.2 & 0.2 & 0.2 & 0.2 & 0.2 \\
\hline Expoente LFS & 0.33 & 0.33 & 0.33 & 0.33 & 0.33 \\
\hline
\end{tabular}

\section{RESULTADOS}

Inicialmente foi escolhida a condição de operação de 3875rpm e torque de $60 \mathrm{Nm}$ e foram feitas as simulações para todos os combustíveis. Em seguida foram feitas as simulações para a condição $3875 \mathrm{rpm}, 105 \mathrm{Nm}$. Os dados necessários de entrada do modelo também incluem informações do combustível, tais como: conteúdo volumétrico do percentual de gasolina, etanol e água; relação ar-combustível (A/C); Poder Calorífico Inferior (PCI); densidade. Todas as informações foram retiradas da tabela 2. Cabe ressaltar que os resultados simulados do modelo de combustão Wiebe foram publicados por Melo et al. [3]

4.1. Resultados para $60 \mathrm{Nm}$.

As comparações dos resultados experimentais (EXP) e simulados (SIM) para a condição de $60 \mathrm{Nm}$ e $3875 \mathrm{rpm}$, tanto para o modelo de Wiebe quanto para o modelo fractal, são apresentadas na tabela 9.

Tabela 9: Resultados simulados (SIM) e experimentais (EXP) de pressão $\left(\mathrm{P}_{\max }\right)$, IMEP e $\mathrm{V}_{\text {air }}$ (vazão de ar) para $3875 \mathrm{rpm}$ a $60 \mathrm{Nm}$.

\begin{tabular}{|l|c|c|c|c|c|}
\hline \multicolumn{1}{|c|}{ Combustível } & H0 & H30 & H50 & H80 & H100 \\
\hline Pado & & & & & \\
\hline Pmax - EXP (MPa) & 2,64 & 3,34 & 3,77 & 4,01 & 4,10 \\
\hline Pmax - SIM Fractal (MPa) & 2,68 & 3,41 & 3,79 & 4,09 & 4,09 \\
\hline Dif - EXP x SIM Wiebe (\%) & 1,5 & 2,1 & 0,5 & 2,0 & $-0,2$ \\
\hline Dif - EXP x SIM Fractal (\%) & 0,4 & $-2,4$ & 0,0 & $-1,7$ & 0,0 \\
\hline \hline IMEP - EXP (MPa) & 0,62 & 0,62 & 0,63 & 0,63 & 0,63 \\
\hline IMEP - SIM - Wibe (MPa) & 0,62 & 0,62 & 0,64 & 0,63 & 0,64 \\
\hline IMEP - SIM Fractal (MPa) & 0,60 & 0,60 & 0,62 & 0,65 & 0,66 \\
\hline Dif - EXP x SIM Wiebe (\%) & 0,0 & 0,0 & 1,6 & 0,0 & 1,6 \\
\hline Dif - EXP x SIM Fractal (\%) & $-3,2$ & $-3,2$ & $-1,6$ & 3,2 & 4,8 \\
\hline \hline Vair - EXP (kg/h) & 98,36 & 92,88 & 95,56 & 98,80 & 101,36 \\
\hline Vair - SIM Wiebe (kg/h) & 24,47 & 23,24 & 23,74 & 24,61 & 25,49 \\
\hline Vair - SIM Fractal (kg/h) & 97,82 & 92,91 & 94,94 & 98,52 & 101,32 \\
\hline Dif - EXP x SIM Wiebe (\%) & $-0,5$ & 0,0 & $-0,6$ & $-0,4$ & 0,6 \\
\hline Dif - EXP x SIM Fractal (\%) & $-0,5$ & 0,0 & $-0,6$ & -0.3 & 0,0 \\
\hline
\end{tabular}


Da tabela 9 pode ser verificado que com relação a pressão máxima e IMEP, as diferenças encontradas entre os resultados experimentais e simulados (Wiebe e Fractal) foram inferiores a 5\%. Para a vazão de ar de admissão as diferenças foram inferiores a $1 \%$. Todas as simulações foram consideradas satisfatórias [2,4].

Nas figuras 3 e 4, para o combustíveis H0 e H50 na condição de operação 3875 rpm e $60 \mathrm{Nm}$, são apresentados gráficos das curvas de pressão: - simuladas pelos modelos fractal (linha pontilhada) e Wiebe (linha tracejada); - experimental (linha contínua). As curvas dos demais combustíveis estão no Anexo I.

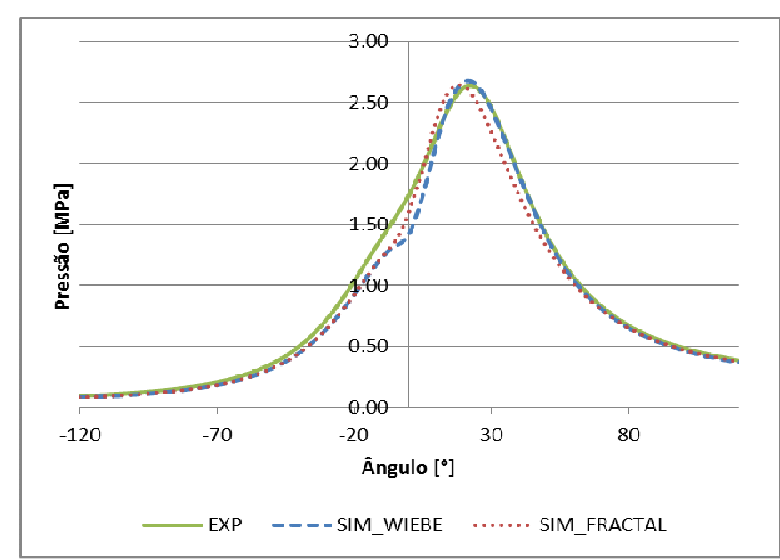

Figura 3: Curva de pressão simulada (Wiebe e fractal) e experimental de $\mathrm{H} 0$ para $3875 \mathrm{rpm}$ a $60 \mathrm{Nm}$

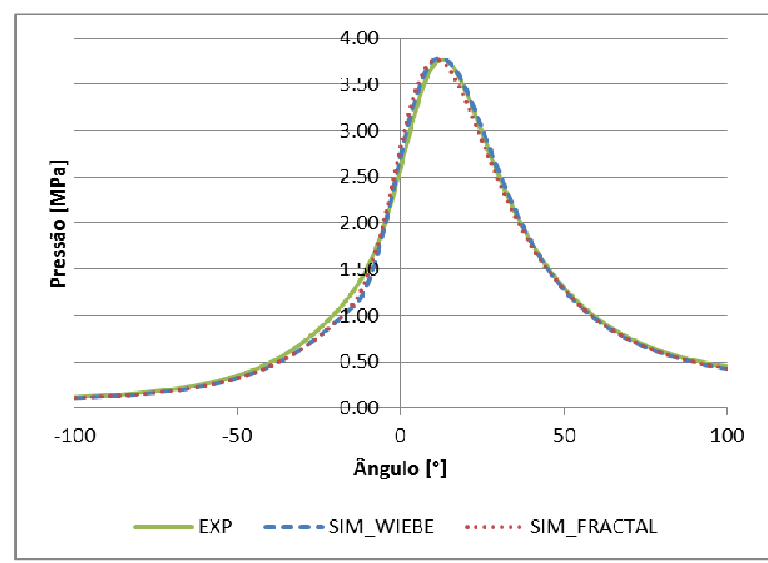

Figura 4: Curva de pressão simulada (Wiebe e fractal) e experimental de $\mathrm{H} 50$ para $3875 \mathrm{rpm}$ a $60 \mathrm{Nm}$

A comparação direta da simulação Wiebe com a simulação fractal mostra que os resultados de pressão máxima $\left(\mathrm{P}_{\max }\right)$ e vazão de ar $\left(\mathrm{V}_{\text {air }}\right)$ são equivalentes. Com relação ao IMEP, apesar de todos os resultados serem inferiores a 5\%, o modelo Wiebe apresentou erros menores que o fractal, porém, dependentes de ajustes específicos para cada combustível em função dos dados experimentais. $\mathrm{O}$ modelo fractal apresentou um caráter preditivo para os combustíveis H30, H50 e H80, após ajustes realizados no modelo para os combustíveis $\mathrm{H} 0$ e H100.

Na tabela 10 é apresentada uma comparação entre os dados experimentais e simulados (Wiebe e Fractal) para o NOx para os diferentes combustíveis, na condição 60Nm, $3875 \mathrm{rpm}$. Os resultados foram obtidos com os parâmetros de ajuste da tabela 11 .

Tabela 10: Resultados de simulação de NOx (Wiebe e fractal) para $3875 \mathrm{rpm}$ a $60 \mathrm{Nm}$.

\begin{tabular}{|c|c|c|c|c|c|}
\hline Combustível & H0 & H30 & H50 & H80 & H100 \\
\hline NOx EXP (ppm) & 2096 & 2638 & 2766 & 2650 & 2521 \\
\hline NOx SIM_Wiebe (ppm) & 2091 & 2615 & 1778 & 2657 & 2511 \\
\hline NOx SIM_Fractal (ppm) & 2096 & 2234 & 2938 & 2901 & 2514 \\
\hline Dif_Wiebe (\%) & $-0,2$ & $-0,9$ & 0,4 & 0,3 & $-0,4$ \\
\hline Dif_Fractal (\%) & 0 & 15 & -6 & -9 & 0 \\
\hline
\end{tabular}

Tabela 11: Parâmetros de ajuste da simulação de NOx para $3875 \mathrm{rpm}$ a $60 \mathrm{Nm}$.

\begin{tabular}{|c|c|c|c|c|c|}
\hline Combustível & H0 & H30 & H50 & H80 & H100 \\
\hline NOx_MULT (Wiebe) & 1 & 1 & 1 & 1 & 1 \\
\hline NOx_POST (Wiebe) & 0,5 & 0,4 & 0,34 & 0,29 & 0,31 \\
\hline NOx_MULT (Fractal) & 1 & 1 & 1 & 1 & 1 \\
\hline NOx_POST (Fractal) & 2.57 & 2.19 & 1.93 & 1.55 & 1.29 \\
\hline
\end{tabular}


A partir dos dados da tabela 10, pode ser verificado, que os resultados das simulações para o modelo de Wiebe foram inferiores a $1 \%$, porém com a necessidade de ajuste individual dos parâmetros do modelo para cada combustível. Na modelagem fractal foram feitos ajustes do modelo apenas para os combustíveis H0 e H100. Para os outros combustíveis foi usada uma equação de regressão linear, dando um caráter preditivo às emissões de NOx. Entretanto, apesar dos erros para os combustíveis H30, H50 e H80 terem sido maiores que os apresentados pelo modelo de Wiebe, os resultados dessas simulações foram preditivos.

Com relação à simulação das emissões de hidrocarboneto e de $\mathrm{CO}$, não foi possível a obtenção de resultados satisfatórios com o modelo fractal, com o uso de diferentes combustíveis. Sendo assim, não serão apresentadas as comparações com o modelo de Wiebe. Segundo Melo et al. [3] foi possível com ajustes específicos a obtenção de erros menores que 5\% para $\mathrm{HC}$ e $\mathrm{CO}$. A exceção foi a simulação de CO com H100 que apresentou um erro elevado.

\subsection{Resultados para $105 \mathrm{Nm}$}

As comparações dos resultados experimentais (EXP) e simulados (SIM) para a condição de $3875 \mathrm{rpm}$ a $105 \mathrm{Nm}$ são apresentadas na tabela 12. Os resultados incluem os modelos de simulação fractal (SIM Fractal) e de Wiebe (SIM Wiebe).

Tabela 12: Resultados simulados (SIM) e experimental (EXP) de $\mathrm{P}_{\max }$, IMEP e $\mathrm{V}_{\text {air }}, 3875 \mathrm{rpm}, 105 \mathrm{Nm}$

\begin{tabular}{|l|c|c|c|c|c|}
\hline \multicolumn{1}{|c|}{ Combustível } & H0 & H30 & H50 & H80 & H100 \\
\hline Pmax - EXP (MPa) & & & & & \\
\hline Pmax - SIM Wiebe (MPa) & 4,85 & 5,21 & 5,88 & 5,99 & 6,22 \\
\hline Pmax - SIM Fractal (MPa) & 4,80 & 4,96 & 5,93 & 6,11 & 6,38 \\
\hline Dif - EXP x SIM Wiebe (\%) & 1,9 & 1,5 & 0,9 & 2,1 & 2,6 \\
\hline Dif - EXP x SIM Fractal (\%) & $-1,0$ & $-4,8$ & $-3,2$ & $-0,8$ & $-1,0$ \\
\hline \hline IMEP - EXP (MPa) & 1,02 & 1,02 & 1,03 & 1,03 & 1,03 \\
\hline IMEP - SIM - Wibe (MPa) & 1,02 & 0,97 & 0,99 & 1,04 & 1,08 \\
\hline IMEP - SIM Fractal (MPa) & 0,98 & 0,96 & 1,00 & 1,05 & 1,09 \\
\hline Dif - EXP x SIM Wiebe (\%) & 0,0 & $-4,9$ & $-3,9$ & 1,0 & 4,8 \\
\hline Dif - EXP x SIM Fractal (\%) & $-3,9$ & -5.9 & -2.9 & 1,9 & 5,8 \\
\hline \hline Vair - EXP (kg/h) & 141,75 & 137,51 & 140,59 & 145,07 & 149,26 \\
\hline Vair - SIM Wiebe (kg/h) & 142,42 & 137,88 & 141,39 & 145,28 & 149,68 \\
\hline Vair - SIM Fractal (kg/h) & 141,96 & 137.81 & 141,39 & 145,34 & 149,76 \\
\hline Dif - EXP x SIM Wiebe (\%) & 0,5 & 0,3 & 0,6 & 0,1 & 0,3 \\
\hline Dif - EXP x SIM Fractal (\%) & 0,1 & 0,2 & 0,6 & 0,2 & 0,3 \\
\hline
\end{tabular}

Da mesma forma que relatado anteriormente para $3875 \mathrm{rpm}$ a $60 \mathrm{Nm}$, os resultados de simulação de condição $3875 \mathrm{rpm}$ a $105 \mathrm{Nm}$ para ambos os modelos (Wiebe e fractal) apresentaram erros menores que 5\% para $\mathrm{P}_{\max }$, IMEO e $\mathrm{V}_{\text {air }}$ quando comparados com os dados experimentais. Cabe ressaltar que os combustíveis H30, H50 e H80 foram simulados de forma preditiva para o modelo fractal. 
Nas figuras 5 e 6 são apresentadas as curvas de pressão experimental (EXP, linha contínua), simulada pelo modelo Wiebe (SIM_Wiebe, linha tracejada) e simulada pelo modelo Fractal (SIM_Fractal, linha pontilhada) referentes aos combustíveis H0 e H50 para $3875 \mathrm{rpm}$ a $105 \mathrm{Nm}$. As curvas dos demais combustíveis estão no Anexo I.

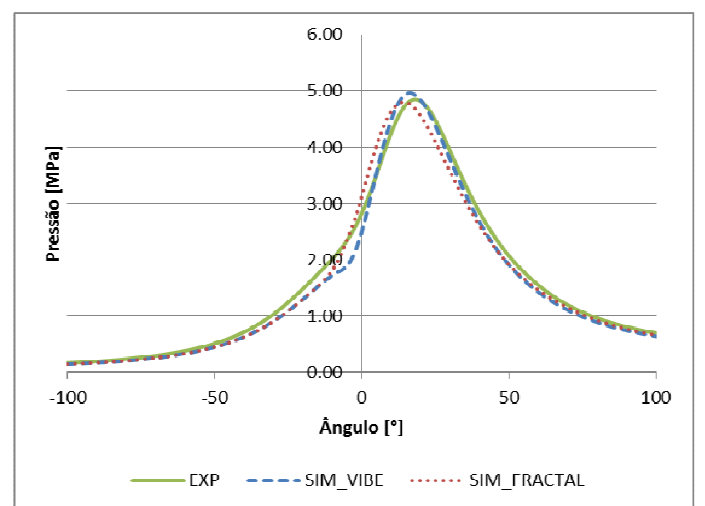

Figura 5: Curva de pressão simulada e experimental de $\mathrm{H} 0$ para $3875 \mathrm{rpm}$ a $105 \mathrm{Nm}$

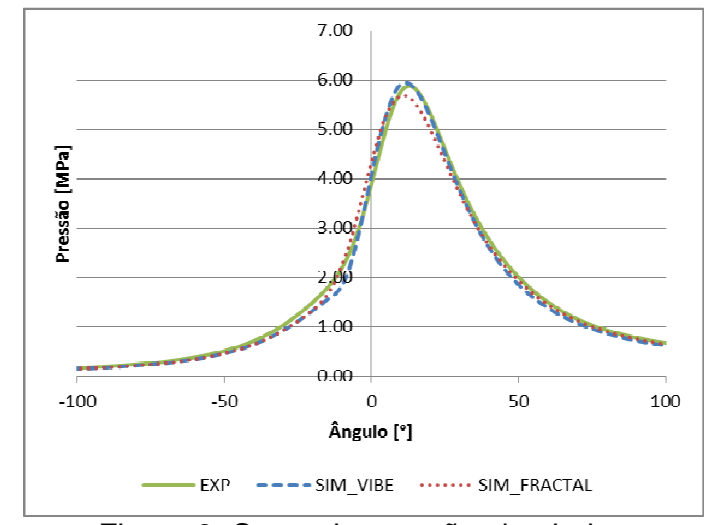

Figura 6: Curva de pressão simulada e experimental de $\mathrm{H} 50$ para $3875 \mathrm{rpm}$ a $105 \mathrm{Nm}$.

Com base nas figuras 5 e 6 pode se constatar que o modelo de Wiebe descreve melhor a curva de pressão experimental após o ponto morto superior devido ao uso de dados experimentais da curva de pressão do motor. O modelo fractal entretanto, além de ser preditivo no caso dos combustíveis H50, consegue um melhor resultado de simulação da curva de pressão no início da combustão, se aproximando mais da curva experimental do que o modelo Wiebe.

Os resultados de emissões de NOx para ambos modelos são apresentados os parâmetros de ajuste para os modelos Wiebe e fractal.

Tabela 12 - Resultados de simulação de NOx (Wiebe e fractal) para 3875 rpm a 105 Nm.

\begin{tabular}{|c|c|c|c|c|c|}
\hline Parâmetro Combustível & H0 & H30 & H50 & H80 & H100 \\
\hline NOX EXP (ppm) & 1940 & 1897 & 1955 & 1892 & 1888 \\
\hline NOX SIM_Wiebe (ppm) & 1938 & 1968 & 1907 & 1980 & 1850 \\
\hline NOX SIM_Fractal (ppm) & 1940 & 1346 & 2244 & 1714 & 1884 \\
\hline Dif_Wiebe (\%) & $-0,1$ & 3,7 & $-2,4$ & 4,7 & -2 \\
\hline Dif_Fractal (\%) & 0 & 29 & -15 & 9 & 0 \\
\hline
\end{tabular}

Table 13 - Parâmetros de ajuste da simulação de NOx para $3875 \mathrm{rpm}$ a 105 Nm.

\begin{tabular}{|l|l|l|l|l|l|}
\hline Parâmetro Combustível & H0 & H30 & H50 & H80 & H100 \\
\hline NOX_MULT (Wiebe) & 1 & 1 & 1 & 1 & 1 \\
\hline NOX_POST (Wiebe) & 1,06 & 0,56 & 0,35 & 0,25 & 0,19 \\
\hline NOX_MULT (Fractal) & 1 & 1 & 1 & 1 & 1 \\
\hline NOX_POST (Fractal) & 3,19 & 2,65 & 2,29 & 1,75 & 1,39 \\
\hline
\end{tabular}

Da tabela 12 pode ser verificado que as simulações de NOx para o modelo Wiebe apresentaram resultados com erro abaixo de 5\% em relação aos dados experimentais, porém com a necessidade de ajustes individuais para cada combustível. Por sua vez, nas simulações utilizando o modelo fractal, apesar de terem apresentado erros superiores em relação aos dados experimentais (máximo de $29 \%$ ), foi possível um ajuste de caráter preditivo para os combustíveis H30, H50 e H80. 
Com relação às emissões de $\mathrm{HC}$ e $\mathrm{CO}$, a exemplo do comentado anteriormente para a condição de torque de $60 \mathrm{Nm}$, não foi possível a obtenção de um modelo preditivo para esses poluentes. Cabe ressaltar que Melo et al.[3] obtiveram resultados de simulação de HC com erros inferiores a 5\%, com o uso do modelo de Wiebe ajustado individualmente para cada combustível, porém com relação ao $\mathrm{CO}$ apenas o combustível H0 apresentou erro abaixo de 5\%. Isso explicita a limitação da modelagem de CO do software utilizado nas simulações com elevados teores de etanol na gasolina. Dessa forma não foi possível a criação de modelo preditivo para as emissões de $\mathrm{CO}$ com o uso da teoria de fractal, uma vez que as equações de formação de CO são as mesmas para ambos os modelos.

\section{CONCLUSÃO}

Esse trabalho apresentou o desenvolvimento de uma modelagem fractal de combustão preditiva para motores FLEX com diferentes teores de etanol na gasolina, não disponível na literatura, com a utilização do software BOOST. Os resultados da simulação do modelo fractal foram comparados a resultados experimentais e aos resultados da simulação usando o modelo Wiebe de duas zonas publicados anteriormente por Melo et al. [3] para duas condições operacionais do motor (60 Nm e 105 Nm @ 3875 rpm).

Os resultados das simulações de emissões de $\mathrm{HC}$ e $\mathrm{CO}$ mostraram que há uma limitação no modelo de emissões do software BOOST para uso preditivo (modelo fractal) com diferentes misturas de etanol na gasolina. Com o uso do modelo de Wiebe e ajustes individuais para cada combustível e condição operacional foi possível se obter erros menores que 5\% nas simulações de HC e CO. As exceções foram o CO para a condição de torque de $60 \mathrm{Nm}$ com o combustível H100 e para a condição de torque de 105 Nm, com os combustíveis H30, H50, H80 e H100.

Com relação às emissões de NOx foi possível a obtenção de um modelo preditivo con erros menores que 15\%, com exceção do combustível H30 na condição de torque $105 \mathrm{Nm}$. Considerando a complexidade dos fenômenos envolvidos na geração de NOx, das mudanças de combustível e o caráter preditivo das simulações, os resultados obtidos podem ser considerados promissores.

Com relação à simulação do desempenho do motor (curva de pressão e pressão média efetiva indicada), todos os erros dos resultados de simulação do modelo fractal foram menores que $5 \%$. Os resultados foram compatíveis com os resultados do modelo Wiebe de duas zonas com a vantagem de apresentarem a característica preditiva, o que o torna uma importante ferramenta para estudos de desempenho de diferentes misturas de etanol na gasolina.

As informações desse artigo podem contribuir para pesquisas na área de modelagem do motor FLEX, bem como dos estudos de desempenho e eficiência desse motor com diferentes misturas de etanol adicionado à gasolina.

Para esclarecimentos adicionais, contatar:

Tadeu Cavalcante Cordeiro de Melo, email: tcm@ petrobras.com.br PETROBRAS/CENPES. Tel: 2121626724. 


\section{REFERÊNCIAS}

[1] MELO, T. C. C., MACHADO, G.B., OLIVEIRA, et al.. "Different Hydrous EthanolGasoline Blends - FTIR Emissions of a Flex-Fuel Engine and Chemical Properties of the Fuels", SAE Technical Paper 2011-36-0080, 2011.

[2] MELO, T. C. C., MACHADO, G. B., MACHADO JR., R. T., et al., "In Cylinder Pressure Curve Simulation On Multifuel Engines - a Comparison Between a Polytrophic and General Thermodynamic Model for Gasoline, Ethanol and Natural Gas", 8th International Conference on Engines for Automobile. SAE 2007-24-0035, Italia, 2007.

[3] MELO, T. C. C., MACHADO, G.B., OLIVEIRA, et al.. "Computer Simulation of a Flex-Fuel Engine Running on Different Gasoline-Hydrous Ethanol Blends", SAE Technical Paper 2012-36-0487, 2012.

[4] HEYWOOD, J. B., 1988, Internal Combustion Engine Fundamentals, 1 ed., McGrawHill. New York, USA.

[5] WOSCHNI, G., "A universally applicable equation for the instantaneous heat transfer coefficient in the internal combustion engine", SAE Technical Papers, 670931, 1967.

[6] WIMMER, A., PIVEC, R., SAMS, T. H., "Heat transfer to the combustion chamber and portwalls of IC engines - Measurement and Prediction", SAE World Congress, 2000-01-0568, Detroit, MI, USA, 2000.

[7] SCHUBERT, C., WIMMER, A., CHMELA, F., "Advanced heat transfer model for CI engines", SAE 2005 World Congress \& Exhibition, 2005-01-0695, Detroit, MI, USA, April, 2005.

[8] AVL, AVL BOOST software version 2013 - Theory, Graz, Austria, 2013

[9] DAMKÖHLER, G., "Der Einfluß der Turbulenz auf die Flammengeschwindigkeit in Gasgemischen", Z. f. Elektroch. 46, No. 11, 601-652, 1940

[10]POULOS S.G., HEYWOOD G.B., "The Effect of Chamber Geometry on SparkIgnition Engine Combustion”, SAE Paper 830334, 1983

[11]NORTH G.L. and SANTAVICCA D.A., "The Fractal Nature of Premixed Turbulent Flames", Comb. Science and Tech., Vol. 72, p.215-232, 1990.

[12]BOZZA, F., GIMELLI, A., MEROLA, S.S., VAGLIECO B.M., "Validation of a Fractal Combustion Model Through Flame Imaging", SAE Technical Paper 2005-011120, 2005.

[13] MAGNUSSEN, B. F., HJERTAGER, B.H., "On Mathematical Modeling of Turbulent Combustion with special emphasis on Soot formation and Combustion", 16th Symposium (International) on Combustion, The Combustion Institute, Pittsburgh, 1976.

[14]PATTAS, K., HAFNER, G.“Stickoxidbildung bei der ottomotorischen Verbrennung“, MTZ, v. 12, pp. 397-404, 1973,.

[15] ONORATI, A., FERRARI, G., D'ERRICO, G., "1D Unsteady Flows with Chemical Reactions in the Exhaust Duct-System of SI Engines: Predictions and Experiments", SAE Technical Paper n. 2001-01-0939

[16] RAGGI, M. V. K., "Modelagem da cinética química de formação de NOx e CO em motores com ignição por centelha", M. Sc. Dissertation, PUC-Minas, Belo Horizonte, MG, Brazil, 2005.

[17]LAVOIE, G. A., BLUMBERG, P. N., "A fundamental model for predicting fuel consumption, $\mathrm{NO}_{\mathrm{x}}$ and $\mathrm{HC}$ emissions of the conventional spark-ignited engine", Combustion Science and Technology, v. 21, pp. 225-258, 1980. 
Anexo I: Curvas de pressão experimental (EXP), Wiebe (SIM_Wiebe) e fractal (SIM_Fractal), torque 60 Nm e 105 Nm @ 3875 rpm.

$\mathrm{H} 30,60 \mathrm{Nm}$

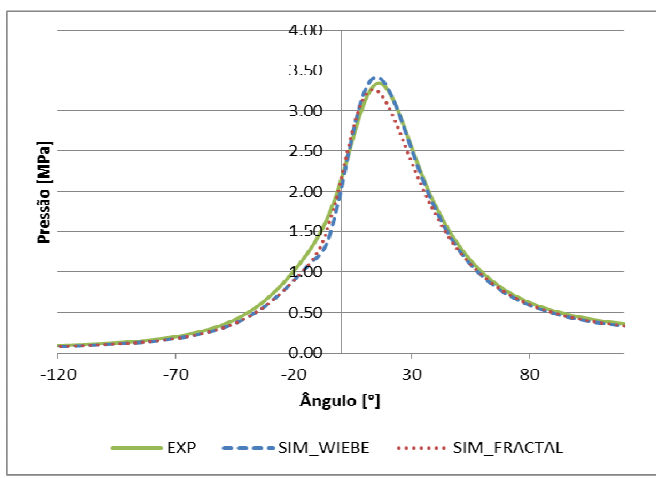

H100, 60Nm

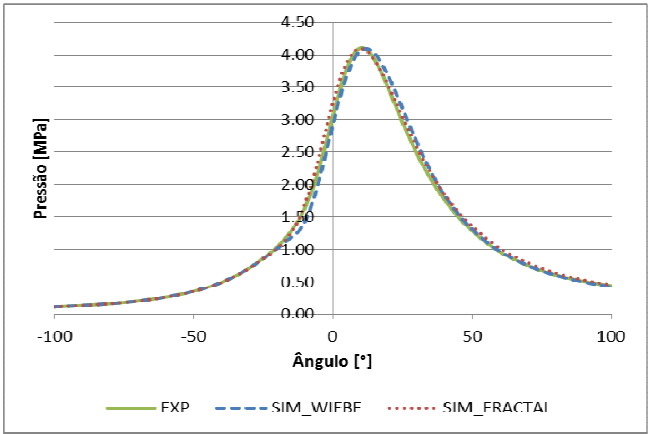

$\mathrm{H} 30,105 \mathrm{Nm}$

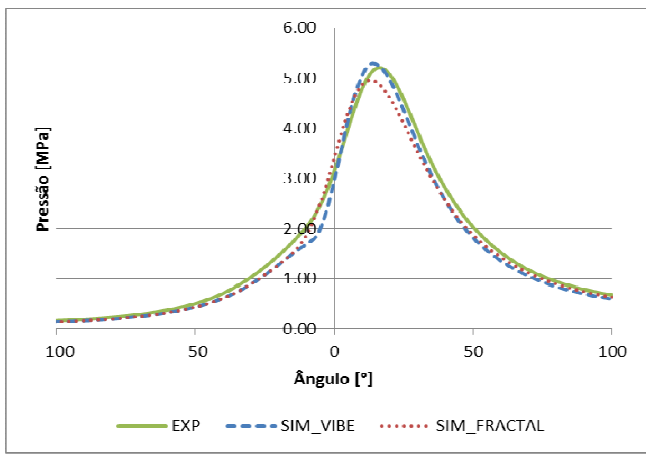

$\mathrm{H} 100,105 \mathrm{Nm}$

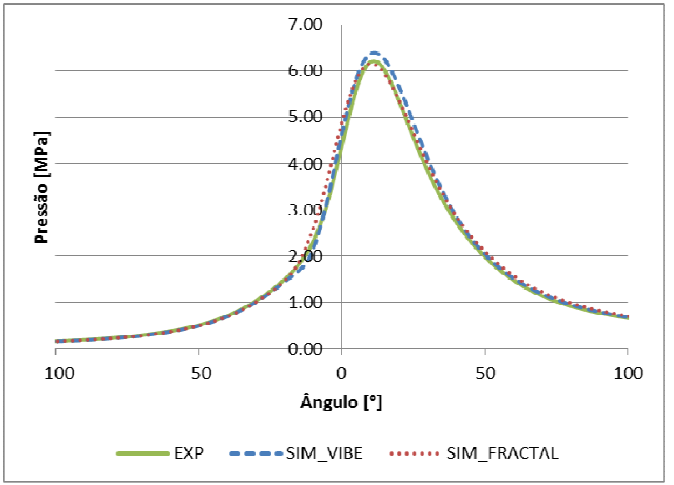

$\mathrm{H} 80,60 \mathrm{Nm}$

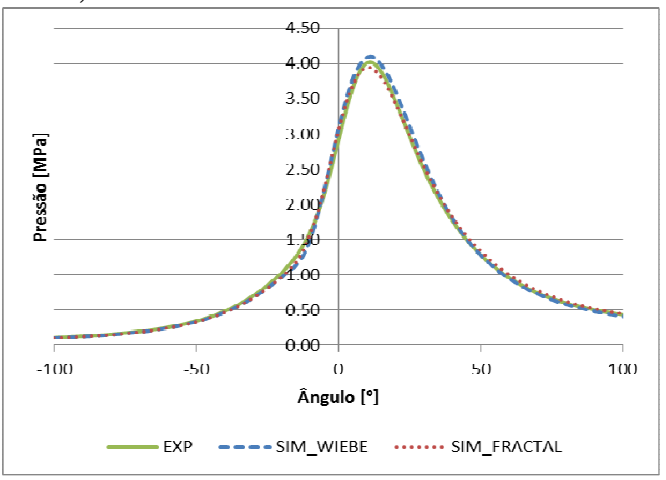

$\mathrm{H} 80,105 \mathrm{Nm}$

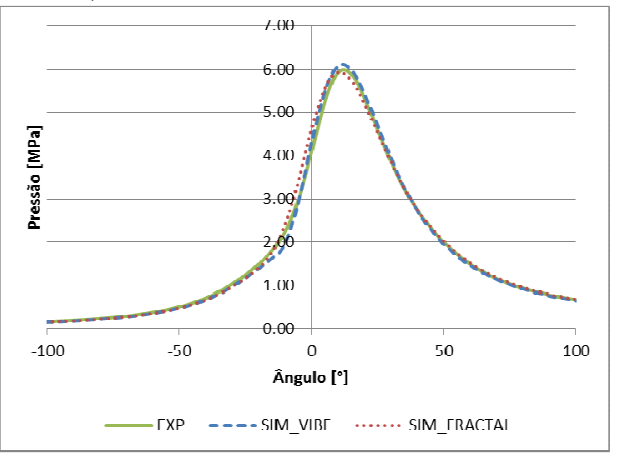

